\title{
Staff Demission Analysis of Public Hospitals - Example of the First Affiliated Hospital of Xi'an Jiaotong University
}

\author{
$\mathrm{Xu} \mathrm{Xu}$
}

The First Affiliated Hospital of Xi'an Jiaotong University, Xi'an, Shaanxi, China

Keywords: Public hospital; Staff demission; Satisfaction.

Abstract. As the foundation of staff demission data from 2013 to 2015 of the First Affiliated Hospital of Xi'an Jiaotong University, through the employee quality control chart, analysis the quit reason, from internal and external for the public hospital staff. Put forward the management policies and suggestions to improve employee job satisfaction and decrease staff demission phenomenon.

\section{公立医院员工离职分析 一以西安交通大学医学院第一附属医院为例 \\ 徐旭 \\ 西安交通大学医学院第一附属医院, 西安, 陕西, 中国}

关键词: 公立医院; 离职; 满意度;

摘要. 以西安交通大学医学院第一附属医院 2013 年至 2015 年员工离职数据为基础, 通过离职 人员质控图, 分析公立医院员工离职内部原因、外部原因。提出相关管理政策和建议, 改善 员工工作满意度, 减少员工离职现象。

引言

随着医疗体制改革深入，行业制度完善，医疗行业人员就业平台进一步扩大。公立医院 从业人员工作机会增多，执业机制趋向社会化。医疗岗位员工面对工作选择机会越来越多， 打破原有体制观念。优秀医生到合资医院、私立医院现象增多; 同时，护理岗位员工寻求新 的择业机会，转行现象增多。本文通过公立医院员工离职质控图分析公立医院员工离职原因， 提出减少公立医院员工离职方案。

研究对象:

本文通过 2013 年 1 月 1 日至 2015 年 12 月 31 日西安交通大学第一附属医院 140 名离职 人员进行统计分析。2014 年离职 79 人, 2015 年离职 61 人。离职人员结构: 医疗岗位人员 29 人，占离职人员 $21 \%$; 护理人员 97 人，占离职人员 $69 \%$; 其它岗位人员 14 人，占离职人 员 $10 \%$ 。

研究目的:

改进公立医院管理方式，提高员工满意度，减少员工离职现象。 研究方法:

对 2013 年 1 月至 2015 年 12 月共计 24 个月离职人数进行统计分析，利用 $\bar{x}-\mathrm{R}$ 控制图分析

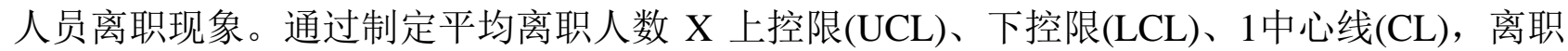
人数极差值 Rs 上控限(UCL)、中心线 $(\mathrm{CL})$, 观察员工离职结构分布, 分析离职原因, 改进管 理方式，制定管理控制图。 
表 1 2013-2015 年员工离职数据表

\begin{tabular}{|c|c|c|c|c|}
\hline 采样号 & 年份 & 月份 & 离职人数 & 移动极差 $\mathrm{R}_{s}$ \\
\hline 1 & 2013 & 1 & 1 & -- \\
\hline 2 & 2013 & 2 & 3 & 2 \\
\hline 3 & 2013 & 3 & 10 & 7 \\
\hline 4 & 2013 & 4 & 6 & 4 \\
\hline 5 & 2013 & 5 & 6 & 0 \\
\hline 6 & 2013 & 6 & 8 & 2 \\
\hline 7 & 2013 & 7 & 9 & 1 \\
\hline 8 & 2013 & 8 & 21 & 12 \\
\hline 9 & 2013 & 9 & 8 & 19 \\
\hline 10 & 2013 & 10 & 3 & 5 \\
\hline 11 & 2013 & 11 & 2 & 1 \\
\hline 12 & 2013 & 12 & 2 & 0 \\
\hline 13 & 2014 & 1 & 3 & 1 \\
\hline 14 & 2014 & 2 & 3 & 0 \\
\hline 15 & 2014 & 3 & 4 & 1 \\
\hline 16 & 2014 & 4 & 6 & 2 \\
\hline 17 & 2014 & 5 & 3 & 3 \\
\hline 18 & 2014 & 6 & 2 & 1 \\
\hline 19 & 2014 & 7 & 17 & 15 \\
\hline 20 & 2014 & 8 & 11 & 6 \\
\hline 21 & 2014 & 9 & 4 & 7 \\
\hline 22 & 2014 & 10 & 6 & 2 \\
\hline 23 & 2012 & 11 & 2 & 4 \\
\hline 24 & 2014 & 12 & 0 & 2 \\
\hline 合计 & & & 140 & 97 \\
\hline
\end{tabular}

平均离职人数: $=\frac{\Sigma x}{n}=5.83$

移动极差均值: $\bar{R}_{\mathrm{s}}=\frac{\Sigma R_{s}}{n-1}=4.21$

查表: $n=2, E 2=2.660$

$$
\mathrm{n}=2 \text {, D4=3.267 }
$$

$\mathrm{X}$ 控制图控制限

中心线: $C L=\bar{X}=5.83$

上控制线: $U C L=\bar{X}+\frac{2}{3} E_{2} \bar{R}_{s}=1329$

下控制线: $L C L=\bar{X}-\frac{2}{3} E_{2} \bar{R}_{s}=1.64$

Rs 控制图控制限

中心线: $C L=\bar{R}_{s}=4.21$ 
上控制线: $U C L=\frac{2}{3} D_{4} \bar{R}_{s}=9.17$

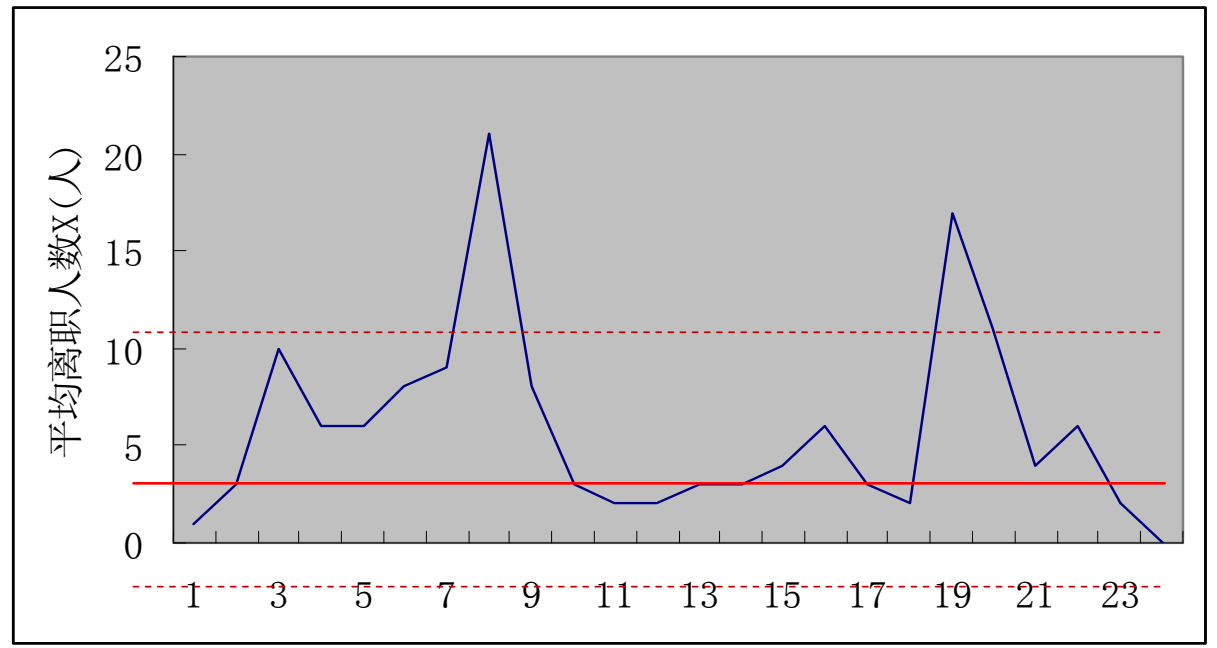

UCL

CL

LCL

图 1: 离职人员 X-R 控制图

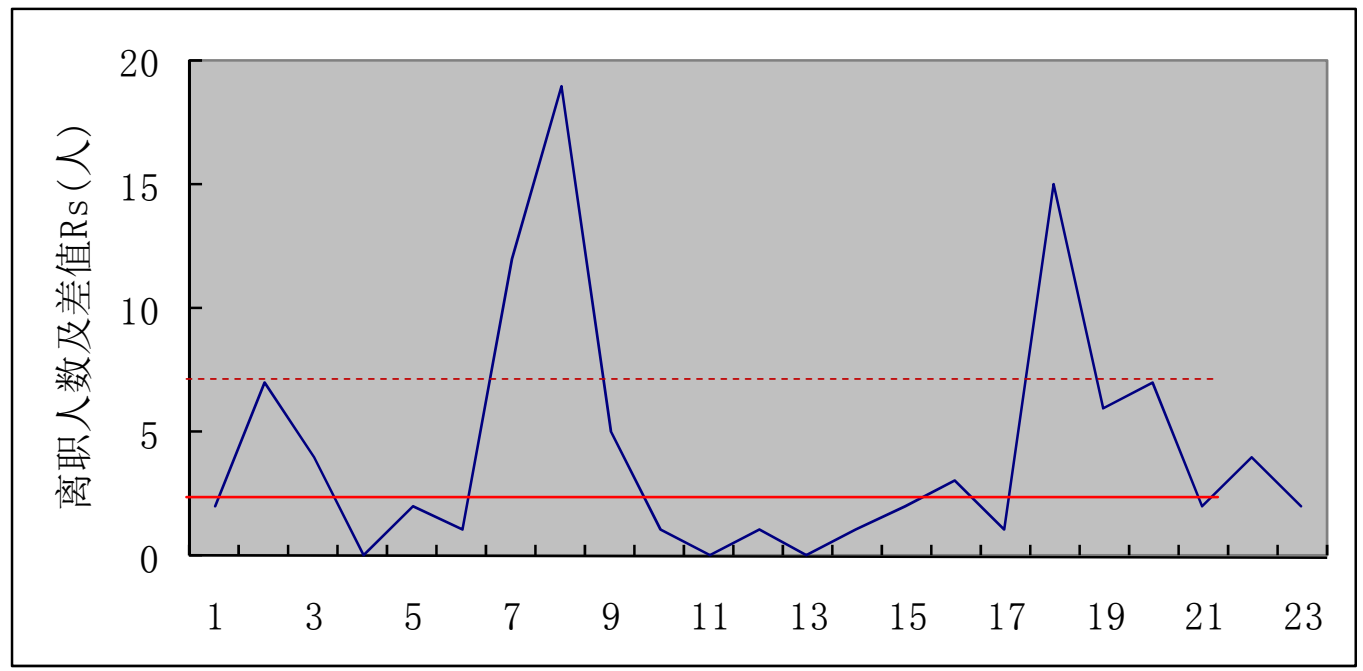

UCL

CL

图 2: 离职人员 X-R 控制图

X 分析质控图中: 8 号、9 号点离职人数超过离职上控制限 13.29(人), 两个点代表时间 分别为 2013 年 8 月、 2014 年 9 月，说明这两个月离职人员激增; $\mathrm{R}_{\mathrm{s}}$ 分析质控图中 8 号点、 9 号点及 19 号点超越控制限 9.17(人)，三个点代表时间分别为 2013 年 8 月、9 月、2014 年 7 月, 说明这三个月与相邻月份离职人数的离散度偏大。一般情况下, $\mathrm{R}_{\mathrm{s}}$ 值超越控制限概率较 小，剔除 8 号点、9 号点及 19 号点，重新计算中心线及控制线。

剔除后 $\mathrm{X}$ 控制限:

中心线: $C L=4.25$

上控制线: UCL $=8.19$

下控制线: $\mathrm{LCL}=0.31$

$\mathrm{R}_{\mathrm{s}}$ 控制限

中心线: $\mathrm{CL}=2.22$.

上控制线: $\mathrm{UCL}=4.84$

每年 7 月、8 月出现离职人数突增, 达到峰值。原因分析：在我院无编制聘用制员工得 到事业编制医院工作机会，造成大量聘用制员工离职。

公立医院员工离职原因错综复杂, 有时离职原因单一, 有时离职原因有几个因素叠加。 
员工通过权衡利弊，最终做出离职决定。

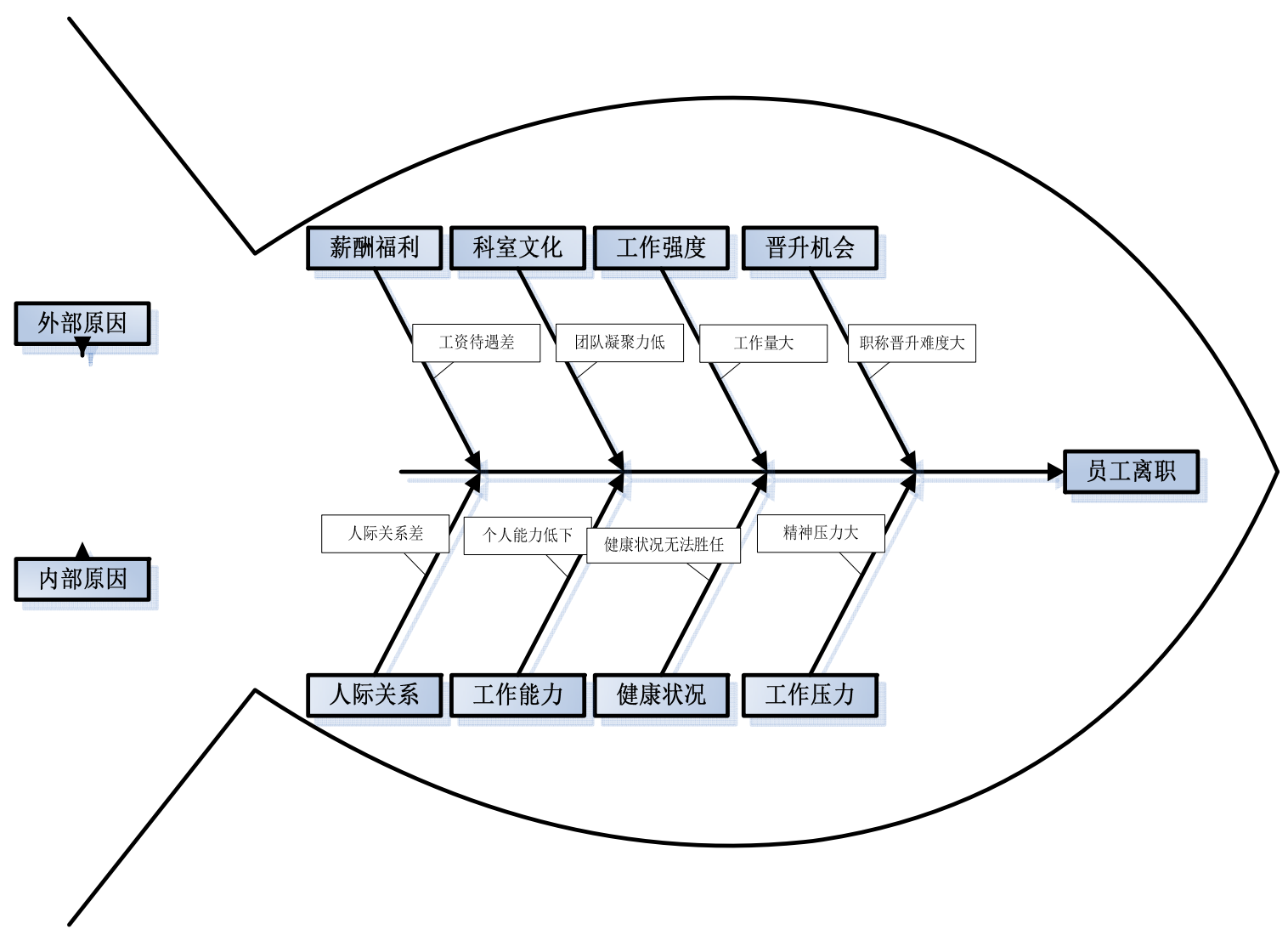

图 3: 员工离职分析图

本文通过鱼骨图将员工离职分为外部原因和内部原因, 根据原因影响程度进行罗列分析, 造成公立医院员工离职外部原因依次为:

1. 医院聘用制员工和编制员工做不到同工同酬，员工福利低于预期，造成员工满意率 低。当外部提供更好的工作机会, 薪酬、福利达到员工心理预期, 离职现象就会出现。医疗 岗位人员离职率居高不下影响医院整个医疗队伍发展, 从战略角度分析对医院学科发展不利。 培养一名优秀医疗人才周期长, 中途流失或是培养完成后流失此类人才，对医疗管理有极大 负面影响 ${ }^{[1][2]}$ 。

2. 科室文化不能深入人心，团队凝聚力低。科室主任、护士长不能从科室员工角度分析 问题，员工情感变化被忽略。

3. 医护人员常年工作量大，员工身体、心理长期承受巨大压力而无法得到释放。造成员 工满意度差，导致员工离职。

4. 职称晋升难度大，尤其护理人员晋升条件高，晋升年限久。

公立医院员工离职外部原因依次为:

1. 员工与科室成员人际关系差, 导致与其他工作人员协调程度低, 沟通不顺畅, 影响双 方工作情绪。

2. 员工自身工作能力低下，无法胜任岗位工作，无法其他工作人员协作，年中考核不合 格, 科室对员工满意度评价低。

3. 员工健康状况低，长期处于身体亚健康状态，继续就职目前岗位工作会存在不安全隐 患。对员工自身、患者都存在潜在危害 ${ }^{[3]}$ 。

4. 员工长期处于高强度工作压力下, 精神状态长期处于高度紧张, 心理压力大, 长期处 于亚健康状态。 
随着国家医疗体制不断完善，为提高医疗水平，使整个社会提高生活水平，打破了公立 医院多年垄断状况, 引入多种医疗竞争机制, 相关政策、制度逐渐向私立医院倾斜。公立医 院为保持优质员工队伍, 适时在管理制度和管理理念进行调整才能保持公立医院目前的行业 优势。

医护人员工作对象是患者, 员工满意度是医疗质量的风向标。医护人员满意度提升，工 作效率高, 医疗质量提升, 患者满意度也随之提升, 随之进入员工管理良性循环模式 ${ }^{[4]}$ 。改 进管理方式，提高员工满意度，减少员工离职现象。

1. 为优秀医技人员提供进修、培训机会

从医院角度出发，挖掘战略性人才，重视员工培养; 从员工角度出发，提高自身技术能 力与竞争力。

医疗岗位人员: 对优秀医疗岗位人员提供保送攻读博士研究生名额, 提供国外一流大学 交流学习机会, 拓宽与国际前沿接轨渠道。推进“青年骨干医师出国研修项目”, 以战略视角 发现培养医疗人才。院内实施多层次、全方位、建立健全住院医师培训制度。以项目研修为 主线, 推进临床高级人才的能力培养与水平提升。护理岗位人员: 鼓励优秀护理人员攻读硕 士学位, 推荐国际一流医院进修及联合培养机会 ${ }^{[5]}$ 。在广泛实施临床护理技能培训的基础上, 推进高级护理项目培训, 提高护理人员工作技能 ${ }^{[6]}$ 。

2. 为优秀员工提供晋升机会

将连续考核成绩优秀、获得相关荣誉、患者满意度高的员工树立典型。同时，将优秀员 工荣誉、表彰经历纳入员工晋升条件中。为医疗岗位人员、护理岗位人员及其他专业技术人 员提供更多晋升机会 ${ }^{[7]}$ 。

3. 提高员工薪酬、福利

通过职工达标大会通过--福利和待遇上做到同工同酬决议，使聘用制员工与正式编制员 工在薪酬、福利上保证一致性。进一步调整薪酬体系, 让薪酬分配更加科学合理, 兼顾到每 位员工的利益。

4. 加强科室文化建设

关心科室员工，及时解决员工工作、生活中的出现的问题。定期开展科室员工生活会， 强调科室柔性化管理。调动员工工作积极性，提升员工归属感 ${ }^{[8]}$ 。

5. 心理疏导缓解员工心理压力

组织对员工自上而下、由医院层面到科室层面的心理疏导, 缓解员工工作压力。对工作 中出现情绪问题和不良医疗事件中分析原因, 制定相关心理疏导课程, 从源头帮助由于心理 压力过大导致出现问题的员工。

\section{References}

[1] LOU Jianhua, ZHU Haiying, XU Lihua, Job Satisfaction of Clinical Nurses, Journal of Nursing Science, vol. 21, No. 3, pp. 46-47, 2006.

[2] GU Songtao, HUNAG Qimin, CHEN Zhiqiang, Research on the job satisfaction of clinical doctors and its significance, Chinese journal of hospital administration,vol.21, No.9, pp.611-614, 2005.

[3] ZHANG Ping, Quit willing of employed nurses, Journal of Clinical and Experimental Medicine, vol.1, No.8, pp 146-147, 2009.

[4] YANG Meiling, WANG Ranran, HOU Shuxiao, Investigation and analysis of relative factors of nurses' quit willing, Modern Nursing, vol. 12, No. 18, pp. 1667-1669, 2006

[5] HUANG Qimen, GU Songtao, CHEN Zhiqiang, A research on Satisfaction Degree of Clinical Doctors in A Grade 3 hospital, Hospital Administration Journal of Chinese People's Liberation Army, vol.9, No.21, pp. 719-722, 2006.

[6] TANG Weiwei, LI Lijuan, HU Bingshui, Causes and its influence factors analysis on staff termination in public hospital --- based on the analysis on 135 cases termination reports in Peking Union Medical College Hospital, Chinese Hospitals, vol.7, No.14, pp. 57-59, 2010. 
[7] GU Songtao, HUANG Qimin, CHEN Zhiqiang, A research on Satisfaction Degree and turnover intention of Clinical Doctors in A Grade 3 hospital, Chinese journal of hospital administration, vol.9, No.22, pp.586-589, 2006.

[8] HUANG Daoyin, Causes Analysis and Countermeasures of Private Hospital Labor Turnover, Journal of traditional Chinese medicine management vol.20, No.1, pp.65-66, 2012. 